\title{
Facile Synthesis of Bisphosphonates
}

\author{
Uma Maheswara Rao KUNDA and Fumio HAMADA* \\ *Department of Applied Chemistry, Graduate School of Engineering \& Resource Science, National University \\ Corporation, Akita University, Akita 010-8502, Japan. \\ E-mail:hamada@gipc.akita-u.ac.jp.
}

\begin{abstract}
Sodium hydride in polyethylene glycol has been used as a sustainable, non-volatile, and ecofriendly catalytic medium for the green synthesis of bisphosphonates with an alkyl/aryl/heterocyclic group fixed at the geminal carbon. All compounds (3a-j) were characterized by IR, ${ }^{1} \mathrm{H}$ and ${ }^{31} \mathrm{P}$ NMR, mass and elemental analysis. The easy recyclability of the reaction medium makes the reaction economically and potentially viable for commercial applications.
\end{abstract}

Key Words : Bisphosphonates, Green Synthesis, NaH in PEG.

\section{INTRODUCTION}

In conjunction with our interest in the development of synthetic methods for the preparation of bisphosphonate derivatives, we have focused our attention to the preparation of new bisphosphonate derivatives by direct insertion of alkyl/aryl/heterocyclic group at the germinal carbon of tetraethyl methylene bisphosphonate in the presence of sodiumhydride in PEG-600 at ambient temperatures. Bisphosphonates are an important class of pharmacologicallyactive molecules, which are widely used in the treatment of various bone diseases such as osteoporosis,[1] Paget's disease,[2] hypercalcaemia[3] and bone metastases secondary to breast cancer,[4] prostate cancer,[5] inhibition of tumor cell proliferation and invasion,[6] inhibition of tumor cell adhesion to bone,[7] inhibition of angiogenesis[8] or induction of apoptosis.[9] Some bisphosphonates also activate the $\gamma \delta$-T cell population, which shows potential cytotoxic activity towards a broad spectrum of tumors.[10] Some others possess anti-inflammatory properties and may be effective in the treatment of rheumatoid arthritis[11] or exhibit anti-parasitic activity.[12]

In the recent years, PEG, a biologically acceptable polymer used extensively in drug delivery and in bioconjugates as tool for diagnostics, emerged as a powerful phase transfer catalyst and performs many useful organic transformations under mild reaction conditions. Moreover, PEG and its monomethyl ethers are inexpensive, thermally stable, recoverable, and are proved to be non-toxic phase-transfer catalysts. The PEG has hitherto not been widely used as a solvent medium but has been used as a support for various transformations.[13] This background prompted to use PEG as reaction medium for this reaction.

In this report, a series of new bisphosphonates with an alkyl/aryl/ heterocyclic group fixed at the geminal carbon was synthesized, using alkyl/aryl/heterocyclic halides and tetraethyl methylene bisphosphonate in the presence of sodium hydride in PEG-600 at ambient temperatures.

\section{EXPERIMENTAL}

\subsection{General}

NMR spectra were recorded on a Bruker instrument at $400 \mathrm{MHz}$ for ${ }^{1} \mathrm{H}$ NMR and $161.5 \mathrm{MHz}$ for ${ }^{31} \mathrm{P}$ NMR in DMSO solution, using TMS as internal standard. Chemical shifts $(\delta)$ are indicated in ppm and coupling constants $(J)$ in Hz. Mass spectra were recorded on ESI-MS mass spectrometer and IR spectra were measured on Perkin-Elmer FT-IR 240-c spectrophotometer using KBr optics. Elemental analyses were performed on a Thermo Finnegan Instrument. All reagents were purchased from Sigma Aldrich and were used without further purification.

\subsection{Synthesis \\ 2.2.1 Typical procedure for the synthesis of Tetraethyl phenylmethylenediphosphonate $(3 \mathrm{~g})$ :}

Tetraethylmethylenebisphosphonate $(0.004 \mathrm{~mol})$ was added drop wise to a dispersion of sodium hydride ( $80 \%$ in mineral oil, $0.004 \mathrm{~mol})$ in $10 \mathrm{~mL}$ of PEG-600, Bromo benzene $(0.004 \mathrm{~mol})$ was added and the mixture was stirred for 2 hours at room temperature. After completion of the reaction as indicated by TLC, diluted $\mathrm{HCl}$ (5\%) was added to the reaction mixture dropwise, and the product were extracted with dichloromethane. Drying extracts on sodium sulfate and evaporation afforded the product. The resulting product was purified by passing it over a silica gel column chromatography with EtOAc / hexane (1:4) as eluent. The remaining mixture was cooled in dry ice-acetone bath to precipitate the PEG and extracted with ether. The ether extract was dried over $\mathrm{MgSO}_{4}$ and evaporated. The recovered PEG has been reused.[13b] The same procedure can be applied for the synthesis of $3 b-j$.

\section{RESULTS AND DISCUSSION}

In an initial endeavor, we carried out the reaction of Bromobenzene $(1 \mathrm{~g})$, Tetraethylmethylenebisphosphonate (2) in the presence of sodium hydride in various solvents at ambient temperature (Table-2). Excellent results were obtained, when the reaction was 
Table 1 Spectral Data of Synthesized compounds (3a-j)

\begin{tabular}{|c|c|c|c|c|c|c|c|}
\hline & \multirow[b]{2}{*}{ Compound Name } & \multicolumn{2}{|l|}{ NMR (DMSO- $\left.d_{6}\right) \delta$} & \multirow{2}{*}{$\begin{array}{l}\mathrm{IR}(\mathrm{KBr}) \\
v_{\max } \mathrm{cm}^{-1} \\
\mathrm{P}=\mathrm{O}, \mathrm{P}-\mathrm{C}\end{array}$} & \multirow{2}{*}{$\begin{array}{c}\text { ESI-MS } \\
\mathbf{m} / \mathbf{z}\left(\mathbf{M}^{+}\right)\end{array}$} & \multicolumn{2}{|c|}{ Elem. Anal. (C\& H) } \\
\hline & & ${ }^{1} \mathbf{H}$ & ${ }^{31} \mathbf{P}$ & & & Calcd. & Found \\
\hline 3a & $\begin{array}{l}\text { Tetraethylquinolin-2-yl } \\
\text { methylenediphosphonate } \\
\qquad\left(\mathrm{C}_{18} \mathrm{H}_{27} \mathrm{NO}_{6} \mathrm{P}_{2}\right)\end{array}$ & $\begin{array}{l}7.54-7.68(\mathrm{~m}, 6 \mathrm{H}), 5.14(\mathrm{~d}, 1 \mathrm{H}, \\
J=9.6 \mathrm{~Hz}), 4.07-3.81(\mathrm{~m}, 8 \mathrm{H}) \\
1.23(\mathrm{t}, 6 \mathrm{H}, J=4.4 \mathrm{~Hz}), 1.01(\mathrm{t}, \\
6 \mathrm{H}, J=6.4 \mathrm{~Hz})\end{array}$ & 29.63 & $\begin{array}{l}1220 \\
755\end{array}$ & 415 & $\begin{array}{c}52.05 \\
6.55\end{array}$ & $\begin{array}{c}52.01 \\
6.53\end{array}$ \\
\hline $3 \mathbf{b}$ & $\begin{array}{l}\text { Tetraethylisoquinolin-1-y } \\
\qquad \begin{array}{l}1 \text { methylene } \\
\text { diphosphonate } \\
\left(\mathrm{C}_{18} \mathrm{H}_{27} \mathrm{NO}_{6} \mathrm{P}_{2}\right)\end{array}\end{array}$ & $\begin{array}{l}7.58-7.74(\mathrm{~m}, 6 \mathrm{H}), 4.93(\mathrm{~d}, 1 \mathrm{H}, \\
J=9.6 \mathrm{~Hz}), 3.87-3.67(\mathrm{~m}, 8 \mathrm{H}), \\
1.21(\mathrm{t}, 6 \mathrm{H}, J=4.0 \mathrm{~Hz}), 1.06(\mathrm{t}, \\
6 \mathrm{H}, J=6.4 \mathrm{~Hz})\end{array}$ & 33.6 & $\begin{array}{l}1234 \\
763\end{array}$ & 415 & $\begin{array}{c}52.05 \\
6.55\end{array}$ & $\begin{array}{c}52.01 \\
6.53\end{array}$ \\
\hline $3 \mathbf{c}$ & $\begin{array}{l}\text { Tetraethyl(2,4-dioxo-1,2, } \\
\text { 3,4-tetrahydropyrimidin- } \\
\text { 5-yl) methylene dipho } \\
\text { sphonate } \\
\left(\mathrm{C}_{13} \mathrm{H}_{24} \mathrm{~N}_{2} \mathrm{O}_{8} \mathrm{P}_{2}\right)\end{array}$ & $\begin{array}{l}6.85(\mathrm{~s}, 1 \mathrm{H}), 6.34(\mathrm{~s}, 1 \mathrm{H}), 5.42(\mathrm{~s}, \\
1 \mathrm{H}), 5.09(\mathrm{~d}, 1 \mathrm{H}, J=9.2 \mathrm{~Hz}) \\
4.05-3.68(\mathrm{~m}, 8 \mathrm{H}), 1.17(\mathrm{t}, 6 \mathrm{H}, J= \\
4.8 \mathrm{~Hz}), 1.06(\mathrm{t}, 6 \mathrm{H}, 4.0 \mathrm{~Hz})\end{array}$ & 31.9 & $\begin{array}{l}1217 \\
812\end{array}$ & 398 & $\begin{array}{c}39.20 \\
6.07\end{array}$ & $\begin{array}{c}39.16 \\
6.02\end{array}$ \\
\hline 3d & $\begin{array}{l}\text { Tetraethyl(6-hydroxy } \\
\text { pyridin-2-yl)methylene } \\
\text { diphosphonate } \\
\left(\mathrm{C}_{14} \mathrm{H}_{25} \mathrm{NO}_{7} \mathrm{P}_{2}\right)\end{array}$ & $\begin{array}{l}6.54(\mathrm{~m}, 3 \mathrm{H}), 5.05(\mathrm{~d}, 1 \mathrm{H}, J=9.4 \\
\mathrm{Hz}), 3.73-4.06(\mathrm{~m}, 8 \mathrm{H}), 1.19(\mathrm{t}, \\
6 \mathrm{H}, J=4.0 \mathrm{~Hz}), 1.08(\mathrm{t}, 6 \mathrm{H}, J= \\
6.8 \mathrm{~Hz})\end{array}$ & 31.6 & $\begin{array}{c}1232 \\
786\end{array}$ & 381 & $\begin{array}{c}44.10 \\
6.61\end{array}$ & $\begin{array}{c}44.03 \\
6.57\end{array}$ \\
\hline $3 e$ & $\begin{array}{l}\text { Tetraethyl (2-amino-6-me } \\
\text { thylpyrimidin-4-yl) } \\
\text { methylenediphosphonate } \\
\qquad\left(\mathrm{C}_{14} \mathrm{H}_{27} \mathrm{~N}_{3} \mathrm{O}_{6} \mathrm{P}_{2}\right)\end{array}$ & $\begin{array}{l}6.58(\mathrm{~s}, 1 \mathrm{H}), 5.62(\mathrm{~s}, 2 \mathrm{H}), 5.13(\mathrm{~d}, \\
1 \mathrm{H}, J=9.4 \mathrm{~Hz}), 4.08-3.76(\mathrm{~m}, \\
8 \mathrm{H}), 2.24(\mathrm{~s}, 3 \mathrm{H}), 1.19(\mathrm{t}, 6 \mathrm{H}, J= \\
4.4 \mathrm{~Hz}), 1.07(\mathrm{t}, 6 \mathrm{H}, J=6.4 \mathrm{~Hz})\end{array}$ & 35.62 & $\begin{array}{c}1225 \\
754\end{array}$ & 395 & $\begin{array}{c}42.53 \\
6.88\end{array}$ & $\begin{array}{c}42.49 \\
6.84\end{array}$ \\
\hline $3 f$ & $\begin{array}{c}\text { Tetraethyl(3-cyano } \\
\text { pyridine-2-yl)methylene } \\
\text { diphosphonate } \\
\left(\mathrm{C}_{15} \mathrm{H}_{24} \mathrm{~N}_{2} \mathrm{O}_{6} \mathrm{P}_{2}\right)\end{array}$ & $\begin{array}{l}7.12-7.17(\mathrm{~m}, 3 \mathrm{H}), 5.10(\mathrm{~d}, 1 \mathrm{H}, \\
J=9.4 \mathrm{~Hz}), 4.07-3.83(\mathrm{~m}, 8 \mathrm{H}) \\
1.13(\mathrm{t}, 6 \mathrm{H}), 1.07(\mathrm{t}, 6 \mathrm{H}, J=6.8 \\
\mathrm{Hz})\end{array}$ & 32.41 & $\begin{array}{c}1232 \\
764\end{array}$ & 390 & $\begin{array}{c}46.16 \\
6.20\end{array}$ & $\begin{array}{r}46.11 \\
6.16\end{array}$ \\
\hline $3 g$ & $\begin{array}{c}\text { Tetraethyl phenyl } \\
\text { methylenediphosphonate } \\
\qquad\left(\mathrm{C}_{15} \mathrm{H}_{26} \mathrm{O}_{6} \mathrm{P}_{2}\right)\end{array}$ & $\begin{array}{l}6.81-6.93(\mathrm{~m}, 5 \mathrm{H}), 4.96(\mathrm{~d}, 1 \mathrm{H}, \\
J=9.4 \mathrm{~Hz}), 4.04-3.69(\mathrm{~m}, 8 \mathrm{H}), \\
1.18(\mathrm{t}, 6 \mathrm{H}, J=4.0 \mathrm{~Hz}), 1.06(\mathrm{t}, \\
6 \mathrm{H}, J=6.4 \mathrm{~Hz})\end{array}$ & 20.63 & $\begin{array}{l}1219 \\
744\end{array}$ & 364 & $\begin{array}{c}49.45 \\
7.19\end{array}$ & $\begin{array}{c}49.39 \\
7.16\end{array}$ \\
\hline $3 \mathbf{h}$ & $\begin{array}{l}\text { Tetraethyl(3-methyl-4-nit } \\
\text { ro phenyl) methylene } \\
\text { diphosphonate } \\
\left(\mathrm{C}_{16} \mathrm{H}_{27} \mathrm{NO}_{8} \mathrm{P}_{2}\right)\end{array}$ & $\begin{array}{l}7.26-7.34(\mathrm{~m}, 3 \mathrm{H}), 5.22(\mathrm{~d}, 1 \mathrm{H}, \\
J=9.6 \mathrm{~Hz}), 3.75-4.07(\mathrm{~m}, 8 \mathrm{H}), \\
1.21(\mathrm{t}, 6 \mathrm{H}, J=5.2 \mathrm{~Hz}), 1.06(\mathrm{t}, \\
6 \mathrm{H}, J=7.2 \mathrm{~Hz})\end{array}$ & 31.52 & $\begin{array}{c}1217 \\
734\end{array}$ & 423 & $\begin{array}{c}45.39 \\
6.43\end{array}$ & $\begin{array}{c}45.36 \\
6.39\end{array}$ \\
\hline $3 \mathbf{i}$ & $\begin{array}{l}\text { Tetraethyl(3-aminopheny } \\
\text { 1) } \\
\text { methylenediphosphonate } \\
\qquad\left(\mathrm{C}_{15} \mathrm{H}_{27} \mathrm{NO}_{6} \mathrm{P}_{2}\right)\end{array}$ & $\begin{array}{l}6.21-6.32(\mathrm{~m}, 4 \mathrm{H}), 5.38(\mathrm{~s}, 2 \mathrm{H}) \\
5.04(\mathrm{~d}, \quad 1 \mathrm{H}, \quad J=9.6 \mathrm{~Hz}) \\
3.76-4.10(\mathrm{~m}, 8 \mathrm{H}), 1.17(\mathrm{t}, 6 \mathrm{H}, J= \\
5.6 \mathrm{~Hz}), 1.11(\mathrm{t}, 6 \mathrm{H}, J=7.2 \mathrm{~Hz})\end{array}$ & 29.61 & $\begin{array}{c}1242 \\
765\end{array}$ & 379 & $\begin{array}{c}47.49 \\
7.17\end{array}$ & $\begin{array}{c}47.45 \\
7.13\end{array}$ \\
\hline $3 \mathbf{j}$ & $\begin{array}{l}\text { Tetraethyl(4-amino-2-nitr } \\
\text { ophenyl)methylene } \\
\text { Diphosphonate } \\
\left(\mathrm{C}_{15} \mathrm{H}_{26} \mathrm{~N}_{2} \mathrm{O}_{8} \mathrm{P}_{2}\right)\end{array}$ & $\begin{array}{l}7.12-7.23(\mathrm{~m}, 3 \mathrm{H}), 5.52(\mathrm{~s}, 2 \mathrm{H}), \\
5.13(\mathrm{~d}, \quad 1 \mathrm{H}, \quad J=9.6 \mathrm{~Hz}) \\
4.09-3.78(\mathrm{~m}, 8 \mathrm{H}), 1.19(\mathrm{t}, 6 \mathrm{H}, J= \\
4.0 \mathrm{~Hz}), 1.05(\mathrm{t}, 6 \mathrm{H}, J=6.4 \mathrm{~Hz})\end{array}$ & 29.37 & $\begin{array}{c}1256 \\
823\end{array}$ & 424 & $\begin{array}{c}42.46 \\
6.18\end{array}$ & $\begin{array}{r}42.41 \\
6.14\end{array}$ \\
\hline
\end{tabular}

carried out in PEG-600.

It was also found that PEG-600 also affects the yield of product. When sodium hydride is replaced with sodium borohydride in the reaction, the products are formed in lower yields ( $>30 \%)$ with longer reaction time (24h). In this reaction, sodium hydride acts as base and abstracts proton from active methylene group of Tetraethylmethylene bisphosphonate, PEG catalyzes by increasing electrophilicity of C-X carbon through a hydrogen bonding by hydroxyl oxygen 
Table 2 Effet of solvents on synthesis of $3 \mathrm{~g}$

\begin{tabular}{cccc}
\hline S. No & Solvent $^{\mathbf{a}}$ & Time (h) & Yield (\%) $^{\mathbf{b}}$ \\
\hline $\mathbf{1}$ & Toluene & 10 & 28 \\
$\mathbf{2}$ & THF & 10 & 19 \\
$\mathbf{3}$ & DMF & 10 & 22 \\
$\mathbf{4}$ & Acetonitrile & 10 & 15 \\
$\mathbf{5}$ & PEG & 2 & 70 \\
\hline
\end{tabular}

${ }^{a}$ Reaction of $1 \mathrm{~g} \& 2$ in the presence of $\mathrm{NaH}$ at $\mathrm{rt}$

${ }^{\mathrm{b}}$ isolated yield

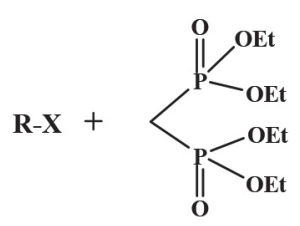

$\mathbf{1} \mathbf{a}-\mathbf{j}$

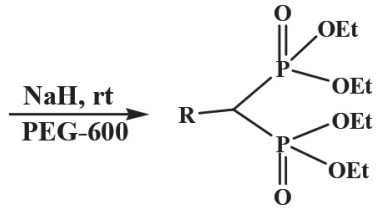

$\mathbf{3} \mathbf{a}-\mathbf{j}$
Scheme-1: Alkylation of Tetraethylmethylene bisphosphonate (3a-j).

with the halide. After this success, The substrate scope of the reaction under the optimized condition was investigated and the results are summarized in Table 2. The reaction was amenable to a wide variety of alkyl/aryl/heterocyclic halides bearing various substituents. The reaction proceeded very smoothly and gave a high yield of the product.

All the products purified by column chromatography and were characterized by elemental analysis, IR, ${ }^{1} \mathrm{H}-,{ }^{31} \mathrm{P}-\mathrm{NMR}$, and MS spectral data it is summarized in Table 1.

The IR spectra of the title compounds (3a-j) showed absorption bands at $1217-1256 \mathrm{~cm}^{-1}(\mathrm{P}=\mathrm{O})$, and $734-823 \mathrm{~cm}^{-1}$ (P-C aliphatic) stretching frequencies.[13c] In the ${ }^{1} \mathrm{H}-\mathrm{NMR}$ spectra of $\mathbf{3 a - j}, \mathrm{P}-\mathrm{C}-\mathrm{H}$ protons appeared as doublets in the region $\delta 4.93-5.22$, due to its coupling with phosphorus. The proton signal of $\mathrm{P}(\mathrm{O}) \mathrm{CH}_{2} \mathrm{CH}_{3}$ showed a multiplet and $\mathrm{P}(\mathrm{O}) \mathrm{CH}_{2} \mathrm{CH}_{3}$ gave a two triplets in the regions of $\delta 3.73-4.10$ and $\delta 1.01-1.23$ respectively.[13c] In mass spectral data, the compounds exhibits their molecular ion peaks at their corresponding values.

\section{CONCLUSIONS}

In this study, $\mathrm{NaH}$ in PEG has been utilized for the efficient one pot synthesis of new bisphosphonate derivatives. The advantages of this procedure are reusability of PEG, operational simplicity and reasonable yields.

\section{References}

[1] Harris, S. T.; Watts, N. B.; Jackson, H.K.; Genant-Wasnish, R. D.; Ross, P.; Miller, P. D.; Licata, A. A.; Chesnut, C. H. "Four-year study of intermittent cyclic etidronate treatment of postmenopausal osteoporosis: Three years of blinded therapy followed by one year of open therapy", Am. J.Med., 95, 557567 (1993).

[2] Miller, P. D., Brown, J. P., Siris, E. S., Hoseyni, M. S., Axelrod, D. W., Bekker, P. J,"A randomized, double-blind comparison of risedronate and etidronate in the treatment of Paget's disease of bone. Paget's Risedronate/Etidronate Study Group." Am J Med., 106 (5), 513-520 (1999).
Table 3 One pot synthesis of bisphosphonate derivatives using NaH/PEG.

1e \& 3e

aisolated yield

[3] Major, P. P., Lortholary, A., Hon, J., Abdi, T., Mills, G., Mensen, H. D., Yunus, F., Bell, R., Body, J., Fehling, E., Seaman, J. "Zoledronic acid is superior to pamidronate in the treatment of hypercalcemia of malignancy: a pooled analysis of two randomized, controlled clinical trials." J Clin Oncol., 19 (2), 558-567 (2001).

[4] Theriault, R. L., Lipton, A., Hortobagyi, G. N., Leff, R., Glück, S., Stewart, J. F., Costello, S., Kennedy, I., Simeone, J., Seaman, J. J., Knight, R. D., Mellars, K., Heffernan, M., Reitsma, D. J. "Pamidronate reduces skeletal morbidity in women with advanced breast cancer and lytic bone lesions: a randomized, placebo-controlled trial. Protocol 18 Aredia Breast Cancer Study Group." J Clin. Oncol, 17 (3), 846-854 (1999).

[5] Sasaki, A., Kitamura, K., Alcalde, R. E., Tanaka, T., Suzuki, A., Etoh, Y., Matsumura, T., "Effect of a newly developed bisphosphonate, YH529, on osteolytic bone metastases in nude mice." Int J Cancer., 77 (2), 279-285 (1998).

[6] Virtanen, S. S, Vaananen, H. K., Harkonen, P. L., Lakkakorpi, P. T., "Alendronate inhibits invasion of PC-3 prostate cancer cells by affecting the mevalonate pathway." Cancer Res., 62 (9), 2708-2714 (2002).

[7] Boissier, S., Magnetto, S., Frappart, L., Cuzin, B., Ebetino, F. H., Delmas, P. D., Clezardin, P., "Bisphosphonates inhibit prostate and breast carcinoma cell adhesion to unmineralized and mineralized bone extracellular matrices." Cancer Res., 57 (18), 3890-3894 (1997).

[8] Hamma-Kourbali, Y., DiBenedetto, M., Ledoux, D., Oudar, O., Leroux, Y., Lecouvey, M., Kraemer, M., "A novel noncontaining-nitrogen bisphosphonate inhibits both in vitro and in vivo angiogenesis." BiochemBiophys Res Commun., 310 (3), 816-823 (2003).

[9] Nishida, S., Kikuichi, S., Haga, H., Yoshioka, S., Tsubaki, M., Fujii, K., Irimajiri, K., "Apoptosis-inducing effect of a new bisphosphonate, YM529, on various hematopoietic tumor cell lines." Biol Pharm Bull., 26 (1), 96-100 (2003).

[10] Miyagawa, F., Tanaka, Y., Yamashita, S., Minato, N., "Essential requirement of antigen presentation by monocyte lineage cells for the activation of primary human gamma delta $\mathrm{T}$ cells by aminobisphosphonate antigen." J Immunol., 166 (9), 5508- 
5514 (2001).

[11] vanOffel, J. F., Shuerwegh, A. J., Bridts, C. H., Bracke, P. G., Stevens, W. J., De Clerck, L. S., "Influence of cyclic intravenous pamidronate on proinflammatorymonocytic cytokine profiles and bone density in rheumatoid arthritis treated with low dose prednisolone and methotrexate." ClinExpRheumatol., 19 (1), 13-20 (2001).

[12] Martin, M. B., Grimley, J. S., Lewis, J. C., Heath, H. T., Bailey, B. N., Kendrick, H., Yardley, V., Caldera, A., Lira, R.,
Urbina, J. A.; Moreno, S. N. J., Docampo, R., Croft, S. L., Oldfield, E., "Bisphosphonates inhibit the growth of Trypanosomabrucei, Trypanosomacruzi, Leishmaniadonovani, Toxoplasma gondii, and Plasmodium falciparum: a potential route to chemotherapy." J Med Chem., 44 (6), 909-916 (2001). [13] Uma MaheswaraRao, K., Jaya Prakash, S., Nayak, S. K., Suresh Reddy, C., "Polyethylene glycol in water: a simple and environment friendly mediumfor C-P bond formation", Catal. Sci. Technol., 1, 1665-1670 (2011). 